\title{
Selectividad por tamaño de las presas en merluza común (Merluccius gayi gayi), zona centro-sur de Chile (1992-1997)
}

\author{
Luis A. Cubillos ${ }^{1}$, Carolina Alarcón ${ }^{1}$ \& Hugo Arancibia ${ }^{2}$ \\ ${ }^{1}$ Laboratorio Evaluación de Poblaciones Marinas (EPOMAR) \\ ${ }^{2}$ Unidad de Tecnología Pesquera (UNITEP), Departamento de Oceanografía \\ Facultad de Ciencias Naturales y Oceanográficas, Universidad de Concepción \\ Casilla 160-C, Concepción, Chile
}

\begin{abstract}
RESUMEN. Se analiza la selectividad por tamaño de las presas en la merluza común (Merluccius gayi gayi) a partir de una base de datos de contenidos estomacales que cubre el periodo 1992-1997. El índice de selectividad de presas de Ursin y los coeficientes de selectividad por tamaño a la edad se calcularon para la propia merluza común (canibalismo), los galatheidos langostino colorado (Pleuroncodes monodon) y langostino amarillo (Cervimunida johni), y los clupeiformes sardina común (Strangomera bentincki) y anchoveta (Engraulis ringens), y otras presas en conjunto. Se confirma que a medida que la merluza crece tiende a consumir presas de mayor tamaño, y que la merluza de más de 2 años tiene preferencia por la fracción juvenil de 0 a 4 años (canibalismo), por anchovetas de 0 y 1 año de edad, y langostinos de grupo de 0 y 1 año de edad. La merluza de más de 4 años presenta igual preferencia por langostino colorado y amarillo de más de 2 años de edad. Otras presas tienen altos coeficientes de selección por los grupos de edad 0 a 4 del predador, las que están representadas principalmente por zooplancton (eufáusidos) y mictófidos. Los coeficientes de selección aquí calculados constituyen una base cuantitativa para avanzar en modelos de evaluación multiespecífico que permitan cuantificar el impacto trófico de merluza común sobre especies de importancia comercial del sistema bento-demersal y pelágico de la zona centro-sur de Chile.
\end{abstract}

Palabras clave: contenido estomacal, predación, coeficientes de conveniencia, índice de selectividad de tamaño, merluza común, Chile central.

\section{Size-based prey selectivity in South Pacific hake (Merluccius gayi gayi), central-southern Chile (1992-1997)}

\begin{abstract}
Size-based prey selectivity in Chilean hake (Merluccius gayi gayi) is analyzed using a data base of stomach contents collected from 1992 to 1997 . The Ursin prey selectivity index and the size-based selectivity coefficients were calculated by age for the Chilean hake (cannibalism); the galatheids squat lobster (Pleuroncodes monodon) and yellow squat lobster (Cervimunida johni); the clupeiforms common sardine (Strangomera bentincki) and anchovy (Engraulis ringens); and other combined prey. Hake are confirmed to consume larger prey as they grow and individuals over two years of age show a preference for the young fraction of the hake population ( $0-4$ years; cannibalism), anchovy (0-1 year), and lobster ( $0-1$ year). Hake over four years of age also prefer squat lobster and yellow squat lobster ( $>2$ years). High selection coefficients are found for the remaining prey, mostly zooplankton (euphausids) and myctophids, with predators from 0 to 4 years. The selection coefficients calculated herein constitute a quantitative basis for improving multispecific evaluation models that allow the quantification of the trophic impact of Chilean hake on commercially important species of the benthic-demersal and pelagic system off central-southern Chile.
\end{abstract}

Key words: stomach content, predation, suitability coefficients, size selectivity index, Chilean hake, central-south Chile.

Autor corresponsal: Luis Cubillos (lucubillos@udec.cl).

\section{INTRODUCCIÓN}

En los ecosistemas marinos, las interacciones tróficas son determinadas por el tamaño de los predadores y sus presas, más bien que por la edad (Floeter \& Temming, 2003). No obstante, varios modelos multiespecíficos como el Análisis de Población Virtual Multiespecífico (MSVPA) son estructurados por edad 
(Beyer \& Sparre, 1983; Magnusson, 1995; Tsou \& Collie, 2001), y se requiere reconciliar con relaciones tróficas basadas en el tamaño de predadores y presas. Una de las formas de análisis que permiten tal reconciliación se basa en los índices de selectividad de presas por tamaño, los cuales son utilizados para determinar matrices de coeficientes de preferencia de presas por edad (Rice et al., 1991; Tsou \& Collie, 2001). En este contexto, el análisis de la selectividad de presas por tamaño permite establecer las bases necesarias para modelar las interacciones tróficas y cuantificar la mortalidad por predación que ejercen los predadores, ya sea en un contexto multiespecífico (Wipple et al., 2000, Livingston \& Jurado-Molina, 2000), o bien monoespecífico considerando explícitamente a la predación (Hollowed et al., 2000).

A nivel mundial, las especies del Género Merluccius han sido consideradas como importantes predadores en el ecosistema que habitan. A su vez, el canibalismo es un fenómeno conocido, así como el impacto trófico sobre otras especies, particularmente de importancia pesquera. Tal es el caso de $M$. productus (Livingston \& Bailey, 1985; Livingston et al., 1986), M. capensis en Sud África (Macpherson \& Roel, 1987; Payne et al., 1987; Punt et al., 1992), M. hubbsi en el Océano Atlántico Suroccidental (Angelescu \& Prenski, 1987), M. gayi peruanus en Perú (Espino \& Wosnitza-Mendo, 1989; Castillo et al., 1989), y M. merluccius en el Mar Cantábrico (Velasco \& Olaso, 1998).

En Chile, la merluza común (Merluccius gayi gayi) constituye uno de los principales recursos explotados por la flota industrial de arrastre (Cubillos et al., 2003a), y uno de los predadores más importantes del sistema bento-demersal y pelágico de la zona centro-sur de Chile (Cubillos et al., 2003b; Neira et al., 2004). En general, la frecuencia de ocurrencia de las presas de merluza común no ha cambiado significativamente entre 1959 y 1987 (Arancibia \& Fuentealba, 1993), siendo las más importantes y persistentes los crustáceos Pterygosquilla armata, langostino colorado (Pleuroncodes monodon), langostino amarillo (Cervimunida johni) y eufáusidos Euphausia mucronata, los peces anchoveta (Engraulis ringens), sardina común (Strangomera bentincki) y la propia merluza común. Cubillos et al. (2003b) analizaron la composición de las especies presa (en peso) entre 1992 y 1998, y encontraron que se compone básicamente de eufáusidos (31,3\%), stomatópoda $P$. armata, galatheidae $P$. monodon y C. johni $(21,9 \%)$, clupeiformes $E$. ringens y $S$. bentincki (11,2\%), y canibalismo (26,4\%). Básicamente, las presas importantes en la dieta de la merluza común, como los langostinos colorado y amarillo, los clupeiformes sardina común y anchoveta, y la propia merluza constituyen recursos pesqueros de importancia en la zona centro-sur y el impacto que podría tener la merluza común sobre ellos requiere ser estudiado.

Aunque en Chile varios estudios han analizado la alimentación de merluza común, e.g. Hermosilla (1959), Bahamonde \& Cárcamo (1959), Hoyl (1967), Arana \& Williams (1970), Gallardo et al. (1980), Meléndez (1984), Arancibia et al. (1986), Arancibia \& Meléndez (1987), Arancibia (1989), Fuentealba (1993), Arancibia \& Fuentealba (1993), Arancibia et al. (1998) y Cubillos et al. (2003b), la mayoría de ellos han sido de carácter descriptivo y muchos no han estado orientados hacia bases cuantitativas que permitan avanzar hacia un modelo multiespecífico tomando en cuenta el tamaño de las presas. Los mayores esfuerzos han estado basados en modelos de balance de masa como ECOPATH with ECOSIM (Christensen \& Pauly, 1992; Walters et al., 1997) por Arancibia et al. (2003) y Neira et al. (2004), quienes describen y analizan la estructura e interacciones tróficas entre las especies comercialmente explotadas en la zona centro-sur. Solamente Jurado-Molina et al. (2006) analizaron la mortalidad por canibalismo de merluza común mediante ecuaciones del MSVPA, y aunque concluyen que la mortalidad por canibalismo es ejercida principalmente por la fracción adulta del stock (cuatro y más años de edad), los coeficientes de conveniencia basados en la selectividad de presas están centrados en sólo dos años de datos.

Con la finalidad de establecer las bases cuantitativas que permitan avanzar hacia un modelo multiespecífico de interacción trófica estructurado por edad, el objetivo de este trabajo es analizar la selectividad por tamaño de las presas de merluza común en el periodo 1992-1997, con énfasis en las presas de importancia comercial, y determinar los coeficientes de selectividad por tamaño y la ración diaria per cápita. Para estimar la razón óptima entre el tamaño del predador y de las presas, se utiliza el índice de selectividad de Ursin (1973), asumiendo que la preferencia de presas por tamaño es independiente del tamaño del predador. La hipótesis de trabajo considera que si existe un tamaño óptimo entre predador y presa, se esperaría que a medida que los ejemplares de merluza común crecen, consumen presas de mayor tamaño corporal, pero en términos absolutos el efecto neto se traduce en la selección de presas 
constituidas principalmente por la fracción más juvenil de las poblaciones presas.

\section{MATERIALES Y MÉTODOS}

Se utilizó una base de datos de contenido estomacal de merluza común que cubre el periodo 1992-1997, y que ha sido documentada en Arancibia et al. (1998). La base de datos fue establecida a partir de muestreos semanales de estómagos en la pesquería industrial de arrastre, que operó sobre la plataforma continental de la zona centro-sur de Chile, principalmente entre los $34^{\circ} \mathrm{S}$ y $40^{\circ} \mathrm{S}$. Los individuos capturados fueron preservados congelados para evitar problemas relacionados con la digestión de las presas (Stobberoup, 1992; Arancibia et al., 1998). A cada espécimen muestreado se registró la longitud total, peso y sexo, y los estómagos fueron preservados congelados. Los estómagos revertidos fueron excluidos del análisis. Todos los ítemes presa fueron identificados a nivel de especie, contados y pesados con una balanza de 0,01 $\mathrm{g}$ de precisión. La importancia de cada ítem presa se calculó considerando el aporte en peso de cada uno respecto del total de presas en los contenidos estomacales.

Para analizar la relación entre el peso de la presa y el tamaño del predador (en peso y longitud) se realizó un análisis exploratorio con modelos aditivos generalizados (GAM). Esta técnica fue utilizada debido a que la relación entre el tamaño del ítem presa y el tamaño del predador puede no ser estrictamente lineal. Se utilizó el paquete computacional "mgcv" (v. 1.1-8) of Wood $(2000,2003)$ para el lenguaje y programa R v. 2.0.2 (Ihaka \& Gentleman, 1996; http://www.r-project.org). La variable dependiente, peso del ítem presa, fue transformada con la familia potencial utilizando un exponente de 0,2. Esta transformación fue necesaria para cumplir con el diagnóstico de los residuales y bondad de ajuste de las relaciones encontradas.

Para establecer la razón óptima entre el peso del predador y el peso de la presa, se utilizó el índice de selección por tamaños de Ursin (1973). En este índice, el tamaño de cada presa en el estómago de cada predador es calculado como la razón entre el peso del predador y el peso de la presa, y cada razón es designada como un valor ISU, y expresada por:

$$
I S U=\log _{e}\left(\frac{w_{x}}{w_{s}}\right)
$$

donde $w_{x}$ es el peso del predador $x(\mathrm{~g}), w_{s}$ es el peso de la presa $s(\mathrm{~g})$ en el estómago del predador $x$. De acuerdo con Clark (1985), $w_{s}$ corresponde al peso de cada item presa en el contenido estomacal de cada predador y no al peso de cada presa individual. Los estadígrafos del ISU son el promedio y la desviación estándar. El promedio del ISU representa el tamaño promedio entre el predador y sus presas, y la desviación estándar es una medida relativa de la selectividad, siendo inversamente proporcional a ésta. En el presente estudio se analizó el ISU para establecer solamente la importancia del canibalismo y la predación sobre langostino colorado, langostino amarillo, sardina común y anchoveta. El resto de los ítemes presas fueron agrupados y denominados como "otras presas". La homogeneidad temporal de la varianza de los valores ISU se analizó mediante el test de Bartlett y la media mediante test de una vía para varianzas no homogéneas.

La ecuación que mide un índice de conveniencia o adecuación de la presa $s$ del grupo de edad $i$ para el predador $x$ del grupo de edad $j, \xi\left(j_{x}, i_{s}\right)$, está dada por (Bogstad et al., 2003):

$$
\xi\left(j_{x}, i_{s}\right)=\alpha_{1}(x, s) \alpha_{2}(x, s) \alpha_{3}\left(j_{x}, i_{s}\right)
$$

donde $\alpha_{1}(x, s)$ describe una medida de "vulnerabilidad general" de la presa $s$ y el predador $x, \alpha_{2}(x, s)$ representa la sobreposición espacial entre predador y presa; y $\alpha_{3}(x, s)$ describe la selección por tamaño del predador $x$ por la presa $s$. Las constantes $\alpha_{1}(x, s)$ y $\alpha_{2}(x, s)$ varían entre 0 y 1 , y su definición es más bien arbitraria. Al considerar los supuestos del Análisis de Población Virtual Multiespecífico (Beyer \& Sparre, 1983; Magnusson, 1995); esto es, que el predador accede a una producción constante de presas, para los propósitos de este estudio, las constantes $\alpha_{1}(x, s)$ y $\alpha_{2}(x, s)$ no fueron modeladas y se fijaron en la unidad. En cambio, la función de selectividad de presas por tamaño se determinó según la siguiente expresión:

$$
\alpha_{3}\left(j_{x}, i_{s}\right)=\exp \left[-\frac{\left(\ln \left(\frac{W_{x, j}}{W_{s, i}}\right)-\eta\right)^{2}}{2 \sigma^{2}}\right]
$$

donde $W_{x, j}$ es el peso promedio del predador $x$ a la edad $j, W_{s, i}$ es el peso promedio de la presa $s$ a la edad $i$. La constante $\eta$ representa la razón de pesos óptima entre la presa y el predador, y $\sigma$ representa la amplitud 
del tamaño de las presas en la dieta del predador. Se consideró el promedio y desviación estándar del ISU para el periodo de estudio, como estimadores de $\eta$ y $\sigma$, y a partir de los cuales se determinó la matriz de selectividad de presas por tamaño a la edad.

En este estudio, se considera que la merluza común es un predador oportunista en el contexto del corto periodo de tiempo que involucra la predación, lo que permite ponderar la función de selectividad de presas por la importancia relativa en peso de cada presa en los contenidos estomacales de merluza común (el predador del sistema en estudio). Los coeficientes de selectividad fueron estandarizados a la unidad y se incorporó el aporte de otras presas en su estimación. Para las presas de importancia comercial, se utilizó el peso promedio a la edad teórica para evaluar la selectividad de presas por tamaño a la edad. Para ello, se consideró desde la edad 0 hasta la edad más vieja que regularmente aparece en el stock explotable de las especies presa. Para la merluza común se utilizó el peso promedio a la edad dado por los parámetros de crecimiento comunicados por Aguayo \& Ojeda (1987), para langostino colorado se utilizó los parámetros de crecimiento estimados por Roa (1993), y para el langostino amarillo los informados por Arancibia et al. (2005). En el caso de sardina común y anchoveta se utilizó el peso promedio a la edad utilizados en modelos de evaluación de stock edad-estructurados (Canales et al. 2004, Canales \& Canales, 2005). El resumen de los pesos promedio a la edad se presenta en la Tabla 1.

Finalmente, se estimó la ración diaria per cápita $\left(R_{x, j}\right)$ que está relacionada directamente con la tasa de crecimiento del predador e inversamente con la eficiencia de conversión de alimento, i.e.

$$
R_{x, j}=\frac{d w / d t}{K_{1, j}} \approx \frac{w_{j+1}-w_{j}}{K_{1, j}}
$$

Para la tasa de crecimiento en peso a la edad, se utilizó la siguiente expresión:

$$
d w / d t=3 k w_{j}\left(\left(W_{\infty} / w_{j}\right)^{1 / 3}-1\right)
$$

donde $k$ y $W_{\infty}$ son parámetros del modelo von Bertalanffy obtenidos de Aguayo \& Ojeda (1987). Para la eficiencia de conversión de alimento (incremento de alimento por alimento ingerido), se utilizó la ecuación comunicada por Pauly (1986) y parametrizada para la merluza común por Cubillos et al. (2003b), i.e.

$$
K_{1, j}=1-\left(w_{j} / W_{\infty}\right)^{\beta}
$$

donde $\beta=0,0581, w_{j}$ es el peso promedio $(\mathrm{g})$ a la edad $j$ y $W_{\infty}$ es el peso asintótico (g).

\section{RESULTADOS}

La base de datos de contenidos estomacales de merluza común contiene 42.997 estómagos de ambos sexos, de los cuales 28.828 estómagos (67\%) presentaron algún tipo de contenido estomacal. El espectro trófico de la merluza común en cuanto a presas que constituyen recursos pesqueros (en peso), corresponde principalmente a canibalismo $(20,7 \%)$, langostino colorado $(9,8 \%)$, langostino amarillo $(6,5 \%)$, sardina común $(3,0 \%)$ y anchoveta $(2,3 \%)$.

El análisis exploratorio entre el peso de la presa y el peso del predador se muestra en las Figuras 1 y 2. Todas las relaciones fueron significativas $(\mathrm{p}<$ $0,05)$, y los residuales presentaron residuos independientes e idénticamente distribuidos (resultados no mostrados). Se observa que aunque las relaciones no son estrictamente lineales, se puede señalar que a medida que los ejemplares crecen, prefieren en general presas de mayor peso. No obstante, se observa tanto en el caso del canibalismo, como en cuanto a las presas langostino colorado, langostino amarillo, anchoveta, y “otras presas", que el tamaño de presas se incrementa linealmente hasta aproximadamente $1500 \mathrm{~g}$ del predador (Fig. 1) o $60 \mathrm{~cm}$ de longitud total (Fig. 2). Posteriormente, las relaciones tienden a estabilizarse debido probablemente al tamaño máximo de las presas analizadas. Sólo la sardina común presenta un comportamiento diferente, ya que en el rango de datos con más información se observa una leve declinación con el tamaño del predador.

El índice de selectividad de presas de Ursin (ISU), por año y por especies-presa, se presenta en la Tabla 2. Al considerar la distribución de frecuencia del ISU para el periodo de tiempo completo (1992-97), se observa que la distribución es sólo aproximadamente normal (Fig. 3). En efecto, para el caso de las presas que constituyen recursos existió una leve asimetría positiva, comparado con el caso de las "otras presas" donde es normal. Probablemente, la tendencia hacia la asimetría está influenciada por las diferencias interanuales observadas en el promedio y la varianza del ISU (Tabla 2). En efecto, el ISU refleja diferencias significativas entre años (Fig. 4) para el caso de la 


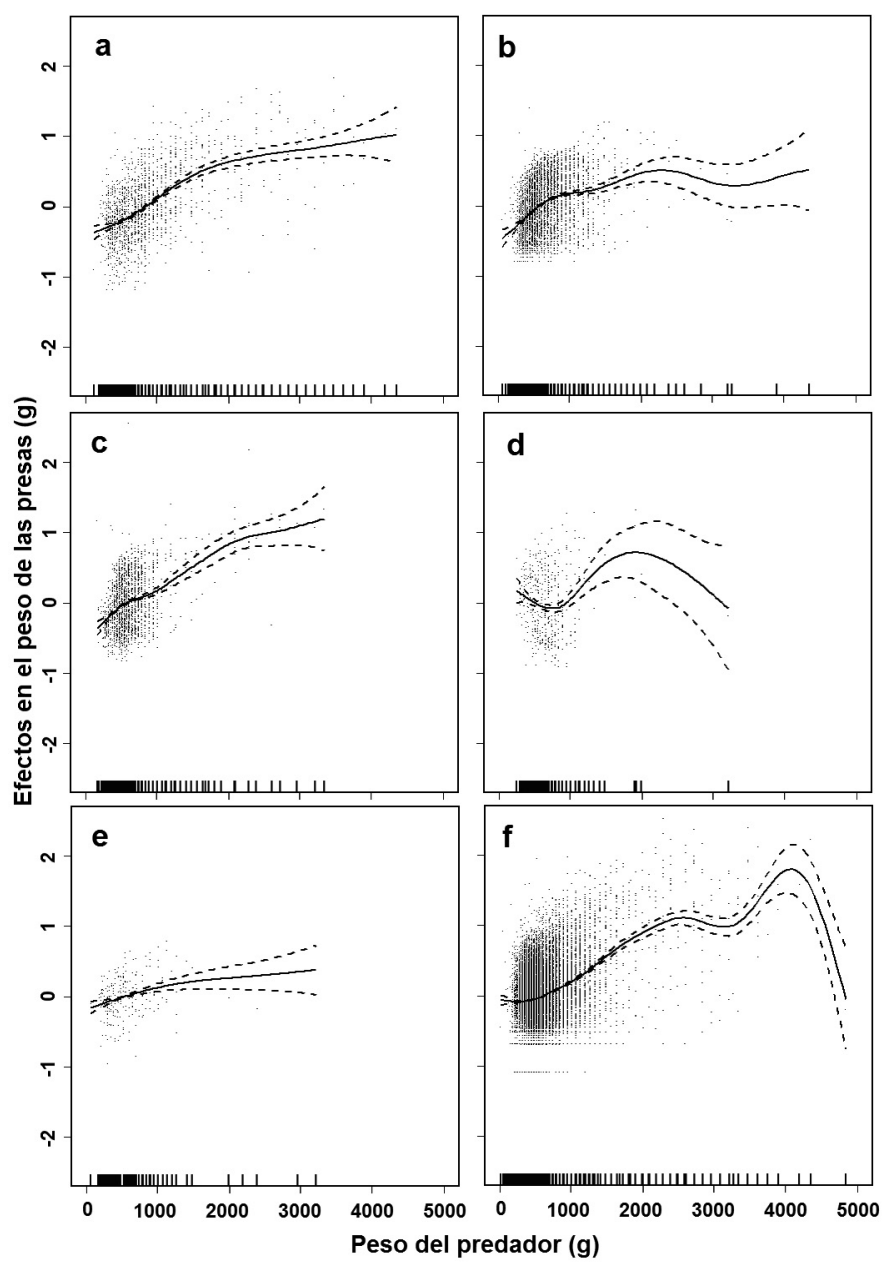

Figura 1. Resultados de los Modelos Aditivos Generalizados (GAM) entre el peso de las presas y el peso del predador (Merluccius gayi gayi) en los contenidos estomacales (1992-1997). a) merluza común (canibalismo), b) langostino colorado, c) langostino amarillo, d) sardina común, e) anchoveta, f) otras presas. En el eje y se indica el suavizador spline utilizado en GAM y representa al peso de las presas en los contenidos estomacales.

Figure 1. Generalised Aditives Models (GAM) results between the preys weight and predator weight (Merluccius gayi gayi) in the stomach contents (1992-1997). a) hake (cannibalism), b) red squat-lobster, c) yellow squat-lobster, d) common sardine, e) anchovy, f) other preys. The y-axis indicates the spline smooth term used in GAM and represents the prey weight in the stomach contents.

merluza común como presa $(\mathrm{F}=14,55 ; \mathrm{p}<0,05)$, langostino colorado $(F=99,91 ; p<0,05)$, sardina común $(\mathrm{F}=54,64 ; \mathrm{p}<0,05)$, anchoveta (test de una vía: $\mathrm{F}=2,88 ; \mathrm{p}<0,05)$, $\mathrm{y}$ otras presas $(\mathrm{F}=73,8 ; \mathrm{p}<$ $0,05)$. Sólo el langostino amarillo no mostró diferencias significativas entre años $(F=2,64 ; p=0,0735)$. A su vez, se detectaron varianzas no homogéneas en todas las presas (test de Bartlett, $p<0,05$ ). Esto indicaría cambios interanuales en el ISU, aspecto que está relacionado con cambios en el peso promedio de las presas como del predador las que quedarían incorporadas en la matriz de selectividad de todo el periodo en términos promedio.

La matriz de los coeficientes de selectividad de alimento para las presas de merluza común (canibalismo), langostino colorado, langostino amarillo, anchoveta y sardina común se estimó considerando el ISU total del periodo de estudio (Tabla 3, Fig. 5). En el caso de la fracción de merluza común que es canibalizada, se observa que los individuos del grupo de edad $0(6,4 \mathrm{~g})$ presentan altos coeficientes de selectividad por merluzas predadoras de 2 a 4 


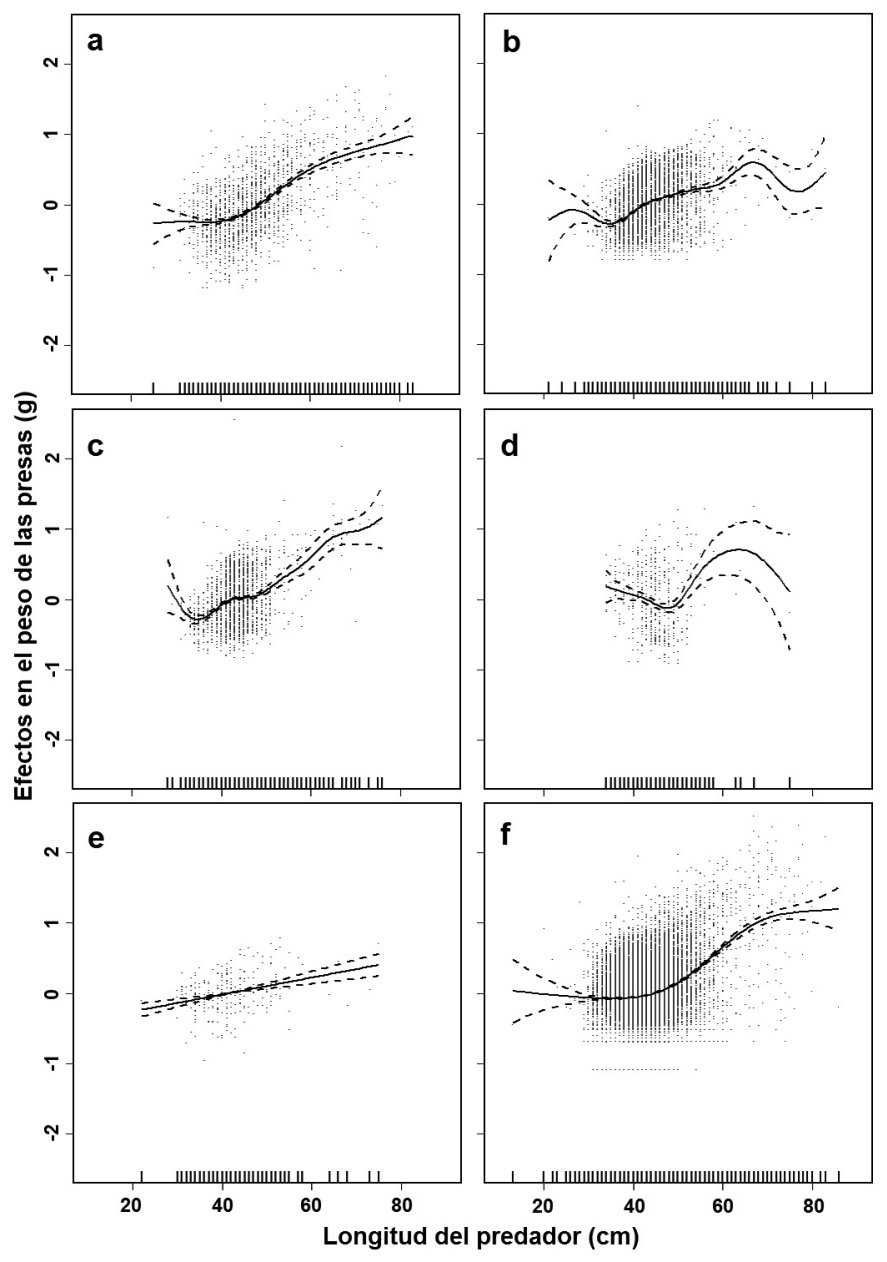

Figura 2. Resultados de los Modelos Aditivos Generalizados (GAM) entre el peso de las presas y la longitud del predador (Merluccius gayi gayi) en los contenidos estomacales (1992-1997). a) merluza común (canibalismo), b) langostino colorado, c) langostino amarillo, d) sardina común, e) anchoveta, f) otras presas. En el eje y se indica el suavizador spline utilizado en GAM y representa al peso de las presas en los contenidos estomacales.

Figure 2. Generalised Aditives Models (GAM) results between the preys weight of predator length (Merluccius gayi gayi) in the stomach contents (1992-1997). a) hake (cannibalism), b) red squat-lobster, c) yellow squat-lobster, d) common sardine, e) anchovy, f) other preys. The y-axis indicates the spline smooth term used in GAM and represents the prey weight in the stomach contents. 
Tabla 1. Peso promedio (g) a la edad de las especies consideradas en el estudio.

Table 1. Average weight (g) at age of the main species considered in the study.

\begin{tabular}{cccccc}
\hline Edad (años) & Merluza común & Langostino colorado & Langostino amarillo & Sardina común & Anchoveta \\
\hline 0 & 6,4 & 0,27 & 0,03 & 6,1 & 6,9 \\
1 & 47,4 & 1,7 & 0,7 & 14,1 & 11,2 \\
2 & 136,9 & 4,3 & 2,8 & 25,6 & 20,5 \\
3 & 273,4 & 7,8 & 6,3 & 34,9 & 29,4 \\
4 & 448,3 & 11,9 & 11,0 & 39,6 & 36,7 \\
5 & 650,7 & 16,1 & 16,5 & - & - \\
6 & 869,9 & 20,3 & 22,5 & - & - \\
7 & 1096,8 & 24,2 & 28,6 & - & - \\
8 & 1323,7 & - & 34,6 & - & - \\
9 & 1544,9 & - & 40,3 & - & - \\
10 & 1756,5 & - & - & - & - \\
11 & 1955,5 & - & - & - & - \\
12 & 2140,5 & - & - & - & - \\
13 & 2310,7 & - & - & - & - \\
14 & 2465,9 & - & - & - & - \\
\hline
\end{tabular}

Tabla 2. Índice de selectividad de presas por tamaño de Ursin (ISU), promedio, desviación estándar (DS) por presa, y número de estómagos de merluza común con cada ítem presa.

Table 2. Ursin's size selectivity prey index (ISU), average, standard deviation (DS), and number of stomachs of hake with each prey item.

\begin{tabular}{|c|c|c|c|c|c|c|c|c|}
\hline \multirow{2}{*}{ Presa } & \multirow{2}{*}{ Estadígrafo } & \multicolumn{6}{|c|}{ Año } & \multirow{2}{*}{ Total } \\
\hline & & 1992 & 1993 & 1994 & 1995 & 1996 & 1997 & \\
\hline \multirow[t]{3}{*}{ Merluza común } & Promedio & 4,17 & 4,18 & 5,14 & 5,86 & 4,8 & 4,37 & 4,34 \\
\hline & DS & 0,97 & 1,28 & 1,84 & 1,9 & 1,91 & 1,73 & 1,38 \\
\hline & $\mathrm{n}$ & 572 & 627 & 45 & 65 & 129 & 64 & 1502 \\
\hline \multirow[t]{3}{*}{ Langostino colorado } & Promedio & 5,25 & 6,49 & 6,65 & 5,88 & 5,35 & 5,61 & 5,8 \\
\hline & DS & 1,43 & 1,73 & 1,49 & 1,55 & 1,73 & 1,43 & 1,64 \\
\hline & $\mathrm{n}$ & 1345 & 771 & 471 & 268 & 253 & 500 & 3608 \\
\hline \multirow[t]{3}{*}{ Langostino amarillo } & Promedio & 5,26 & - & - & - & 5,62 & 5,52 & 5,55 \\
\hline & DS & 1,43 & - & - & - & 1,64 & 1,41 & 1,51 \\
\hline & $\mathrm{n}$ & 87 & - & - & - & 740 & 972 & 1799 \\
\hline \multirow[t]{3}{*}{ Sardina común } & Promedio & 3,58 & 3,33 & 3,33 & 3,80 & 5,75 & 4,31 & 5,01 \\
\hline & DS & 0,64 & 1,54 & 0,58 & 1,06 & 1,79 & 1,45 & 1,83 \\
\hline & $\mathrm{n}$ & 41 & 9 & 22 & 16 & 258 & 87 & 346 \\
\hline \multirow[t]{3}{*}{ Anchoveta } & Promedio & 3,71 & 3,24 & 3,48 & 3,77 & 3,45 & - & 3,66 \\
\hline & DS & 1,02 & 0,42 & 0,68 & 0,88 & 1,09 & - & 0,95 \\
\hline & $\mathrm{n}$ & 140 & 10 & 27 & 50 & 14 & - & 241 \\
\hline \multirow[t]{3}{*}{ Otras presas } & Promedio & 5,84 & 6,2 & 6,27 & 6,17 & 6,47 & 6,66 & 6,21 \\
\hline & DS & 1,90 & 1,94 & 1,85 & 1,74 & 1,69 & 1,55 & 1,82 \\
\hline & $\mathrm{n}$ & 3937 & 5295 & 4212 & 3462 & 2778 & 1648 & 21332 \\
\hline
\end{tabular}


Tabla 3. Coeficientes de selectividad de alimento de merluza común como principal predador de merluza (canibalismo), langostino colorado, langostino amarillo, sardina común, anchoveta y otro alimento (1992-1997).

Table 3. Selectivity coefficients of food for Chilean hake as the main predator on hake (M. común), red squatlobster (L. colorado), yellow squat-lobster (L. amarillo), common sardine (S. común), anchovy (Anchoveta), and other food (Otro alimento) in the period 1992-1997.

\begin{tabular}{|c|c|c|c|c|c|c|c|c|c|c|c|c|c|c|c|c|}
\hline \multirow[b]{2}{*}{ Presa } & \multirow[b]{2}{*}{ Edad } & \multicolumn{15}{|c|}{ Edad predador } \\
\hline & & 0 & 1 & 2 & 3 & 4 & 5 & 6 & 7 & 8 & 9 & 10 & 11 & 12 & 13 & 14 \\
\hline \multirow[t]{15}{*}{ M. común } & 0 & 0.0022 & 0.0537 & 0.1027 & 0.1116 & 0.1042 & 0.0928 & 0.0817 & 0.0721 & 0.0641 & 0.0575 & 0.0522 & 0.0479 & 0.0443 & 0.0414 & 0.0390 \\
\hline & 1 & 0 & 0.0016 & 0.0093 & 0.0210 & 0.0331 & 0.0436 & 0.0522 & 0.0587 & 0.0637 & 0.0673 & 0.0699 & 0.0718 & 0.0731 & 0.0741 & 0.0747 \\
\hline & 2 & 0 & 0.0001 & 0.0011 & 0.0037 & 0.0077 & 0.0124 & 0.0175 & 0.0224 & 0.0270 & 0.0311 & 0.0347 & 0.0378 & 0.0405 & 0.0428 & 0.0448 \\
\hline & 3 & 0 & 0 & 0.0002 & 0.0009 & 0.0022 & 0.0040 & 0.0062 & 0.0087 & 0.0112 & 0.0137 & 0.0160 & 0.0181 & 0.0201 & 0.0218 & 0.0233 \\
\hline & 4 & 0 & 0 & 0 & 0.0003 & 0.0007 & 0.0015 & 0.0026 & 0.0038 & 0.0051 & 0.0065 & 0.0079 & 0.0092 & 0.0104 & 0.0115 & 0.0125 \\
\hline & 5 & 0 & 0 & 0 & 0.0000 & 0.0003 & 0.0007 & 0.0012 & 0.0019 & 0.0026 & 0.0034 & 0.0042 & 0.0050 & 0.0058 & 0.0066 & 0.0072 \\
\hline & 6 & 0 & 0 & 0 & 0 & 0.0001 & 0.0003 & 0.0006 & 0.0010 & 0.0015 & 0.0020 & 0.0025 & 0.0030 & 0.0035 & 0.0040 & 0.0045 \\
\hline & 7 & 0 & 0 & 0 & 0 & 0.0 & 0.0002 & 0.0004 & 0.0006 & 0.0009 & 0.0012 & 0.0016 & 0.0019 & 0.0023 & 0.0026 & 0.0029 \\
\hline & 8 & 0 & 0 & 0 & 0 & 0.0 & 0.0001 & 0.0002 & 0.0004 & 0.0006 & 0.0008 & 0.0011 & 0.0013 & 0.0016 & 0.0018 & 0.0021 \\
\hline & 9 & 0 & 0 & 0 & 0 & 0.0 & 0 & 0.0002 & 0.0003 & 0.0004 & 0.0006 & 0.0008 & 0.0010 & 0.0011 & 0.0013 & 0.0015 \\
\hline & 10 & 0 & 0 & 0 & 0 & 0.0 & 0 & 0.0001 & 0.0002 & 0.0003 & 0.0004 & 0.0006 & 0.0007 & 0.0009 & 0.0010 & 0.0012 \\
\hline & 11 & 0 & 0 & 0 & 0 & 0.0 & 0 & 0 & 0.0001 & 0.0002 & 0.0003 & 0.0004 & 0.0006 & 0.0007 & 0.0008 & 0.0009 \\
\hline & 12 & 0 & 0 & 0 & 0 & 0.0 & 0 & 0 & 0.0001 & 0.0002 & 0.0003 & 0.0004 & 0.0005 & 0.0006 & 0.0007 & 0.0008 \\
\hline & 13 & 0 & 0 & 0 & 0 & 0.0 & 0 & 0 & 0.0000 & 0.0002 & 0.0002 & 0.0003 & 0.0004 & 0.0005 & 0.0006 & 0.0006 \\
\hline & 14 & 0 & 0 & 0 & 0 & 0.0 & 0 & 0 & 0 & 0.0001 & 0.0002 & 0.0003 & 0.0003 & 0.0004 & 0.0005 & 0.0006 \\
\hline \multirow[t]{8}{*}{ L. colorado } & 0 & 0.0387 & 0.0983 & 0.0721 & 0.0461 & 0.0308 & 0.0218 & 0.0164 & 0.0128 & 0.0104 & 0.0087 & 0.0074 & 0.0065 & 0.0058 & 0.0053 & 0.0049 \\
\hline & 1 & 0.0036 & 0.0348 & 0.0520 & 0.0528 & 0.0491 & 0.0447 & 0.0406 & 0.0371 & 0.0342 & 0.0317 & 0.0296 & 0.0279 & 0.0264 & 0.0252 & 0.0242 \\
\hline & 2 & 0.0006 & 0.0125 & 0.0271 & 0.0351 & 0.0388 & 0.0402 & 0.0405 & 0.0401 & 0.0394 & 0.0386 & 0.0377 & 0.0369 & 0.0361 & 0.0354 & 0.0347 \\
\hline & 3 & 0.0002 & 0.0055 & 0.0150 & 0.0227 & 0.0281 & 0.0316 & 0.0340 & 0.0355 & 0.0363 & 0.0368 & 0.0371 & 0.0371 & 0.0371 & 0.0370 & 0.0368 \\
\hline & 4 & 0.0000 & 0.0028 & 0.0092 & 0.0155 & 0.0206 & 0.0247 & 0.0277 & 0.0300 & 0.0317 & 0.0329 & 0.0338 & 0.0344 & 0.0349 & 0.0352 & 0.0354 \\
\hline & 5 & 0 & 0.0017 & 0.0062 & 0.0112 & 0.0158 & 0.0198 & 0.0230 & 0.0255 & 0.0275 & 0.0291 & 0.0303 & 0.0312 & 0.0320 & 0.0325 & 0.0330 \\
\hline & 6 & 0 & 0.0011 & 0.0044 & 0.0086 & 0.0127 & 0.0163 & 0.0194 & 0.0220 & 0.0242 & 0.0259 & 0.0273 & 0.0284 & 0.0293 & 0.0300 & 0.0306 \\
\hline & 7 & 0 & 0.0008 & 0.0034 & 0.0070 & 0.0106 & 0.0140 & 0.0169 & 0.0195 & 0.0216 & 0.0234 & 0.0249 & 0.0260 & 0.0270 & 0.0278 & 0.0285 \\
\hline \multirow[t]{10}{*}{ L. amarillo } & 0 & 0.0946 & 0.0363 & 0.0088 & 0.0026 & 0.0010 & 0.0005 & 0.0002 & 0.0001 & 0.0000 & 0 & 0 & 0 & 0 & 0 & 0 \\
\hline & 1 & 0.0076 & 0.0464 & 0.0485 & 0.0375 & 0.0281 & 0.0215 & 0.0170 & 0.0138 & 0.0115 & 0.0099 & 0.0086 & 0.0076 & 0.0069 & 0.0063 & 0.0058 \\
\hline & 2 & 0.0007 & 0.0139 & 0.0270 & 0.0313 & 0.0314 & 0.0299 & 0.0280 & 0.0261 & 0.0243 & 0.0228 & 0.0214 & 0.0202 & 0.0193 & 0.0184 & 0.0177 \\
\hline & 3 & 0.0001 & 0.0046 & 0.0129 & 0.0192 & 0.0229 & 0.0249 & 0.0259 & 0.0262 & 0.0261 & 0.0258 & 0.0254 & 0.0249 & 0.0245 & 0.0241 & 0.0237 \\
\hline & 4 & 0 & 0.0018 & 0.0066 & 0.0116 & 0.0157 & 0.0187 & 0.0208 & 0.0222 & 0.0232 & 0.0238 & 0.0242 & 0.0244 & 0.0245 & 0.0245 & 0.0245 \\
\hline & 5 & 0 & 0.0008 & 0.0037 & 0.0074 & 0.0109 & 0.0139 & 0.0163 & 0.0181 & 0.0195 & 0.0206 & 0.0214 & 0.0220 & 0.0225 & 0.0228 & 0.0230 \\
\hline & 6 & 0 & 0.0004 & 0.0023 & 0.0050 & 0.0079 & 0.0105 & 0.0129 & 0.0148 & 0.0164 & 0.0176 & 0.0186 & 0.0194 & 0.0201 & 0.0206 & 0.0210 \\
\hline & 7 & 0 & 0.0003 & 0.0015 & 0.0036 & 0.0059 & 0.0083 & 0.0104 & 0.0123 & 0.0138 & 0.0151 & 0.0162 & 0.0171 & 0.0178 & 0.0184 & 0.0189 \\
\hline & 8 & 0 & 0.0002 & 0.0011 & 0.0027 & 0.0047 & 0.0067 & 0.0086 & 0.0104 & 0.0119 & 0.0132 & 0.0143 & 0.0152 & 0.0160 & 0.0166 & 0.0171 \\
\hline & 9 & 0 & 0.0001 & 0.0008 & 0.0021 & 0.0038 & 0.0056 & 0.0073 & 0.0089 & 0.0104 & 0.0116 & 0.0127 & 0.0136 & 0.0144 & 0.0151 & 0.0156 \\
\hline \multirow[t]{5}{*}{ S. común } & 0 & 0.0024 & 0.0193 & 0.0291 & & 0.0307 & & 0.0282 & 0.0269 & 0.0258 & 0.0247 & 0.0238 & 0.0230 & 0.0223 & 0.0217 & 0.0212 \\
\hline & 1 & 0.0006 & 0.0082 & 0.0162 & 0.0207 & 0.0231 & 0.0244 & 0.0251 & 0.0254 & 0.0255 & 0.0254 & 0.0253 & 0.0251 & 0.0249 & 0.0247 & 0.0245 \\
\hline & 2 & 0.0002 & 0.0040 & 0.0094 & 0.0136 & 0.0166 & 0.0187 & 0.0203 & 0.0214 & 0.0222 & 0.0228 & 0.0232 & 0.0235 & 0.0237 & 0.0238 & 0.0239 \\
\hline & 3 & 0.0001 & 0.0026 & 0.0068 & 0.0105 & 0.0134 & 0.0157 & 0.0174 & 0.0188 & 0.0198 & 0.0206 & 0.0213 & 0.0217 & 0.0221 & 0.0224 & 0.0226 \\
\hline & 4 & 0.0000 & 0.0022 & 0.0059 & 0.0094 & 0.0122 & 0.0145 & 0.0163 & 0.0177 & 0.0188 & 0.0197 & 0.0204 & 0.0209 & 0.0213 & 0.0216 & 0.0219 \\
\hline \multirow[t]{5}{*}{ Anchoveta } & 0 & 0 & 0.0133 & 0.0386 & 0.0386 & 0.0286 & 0.0195 & 0.0131 & 0.0091 & 0.0064 & 0.0047 & 0.0036 & 0.0028 & 0.0023 & 0.0019 & 0.0016 \\
\hline & 1 & 0 & 0.0047 & 0.0237 & 0.0343 & 0.0330 & 0.0274 & 0.0216 & 0.0168 & 0.0132 & 0.0106 & 0.0086 & 0.0072 & 0.0061 & 0.0052 & 0.0046 \\
\hline & 2 & 0 & 0.0009 & 0.0089 & 0.0205 & 0.0275 & 0.0293 & 0.0281 & 0.0256 & 0.0228 & 0.0202 & 0.0179 & 0.0160 & 0.0145 & 0.0132 & 0.0121 \\
\hline & 3 & 0 & 0.0003 & 0.0041 & 0.0124 & 0.0203 & 0.0252 & 0.0271 & 0.0271 & 0.0260 & 0.0245 & 0.0229 & 0.0214 & 0.0200 & 0.0188 & 0.0177 \\
\hline & 4 & 0 & 0.0001 & 0.0024 & 0.0085 & 0.0157 & 0.0213 & 0.0247 & 0.0261 & 0.0262 & 0.0257 & 0.0248 & 0.0238 & 0.0227 & 0.0217 & 0.0208 \\
\hline \multicolumn{2}{|c|}{ Otro alimento } & 3480 & 0.6267 & 0.4386 & 0.3408 & 0.2917 & 0.2650 & 0.2492 & 0.2393 & 0.2326 & 0.2279 & 0.2245 & 0.2220 & 0.2200 & 0.2184 & 0.2172 \\
\hline & Total & 1 & 1 & 1 & 1 & 1 & 1 & 1 & 1 & 1 & 1 & 1 & 1 & 1 & 1 & \\
\hline
\end{tabular}

años de edad, para posteriormente disminuir con la edad del predador. El grupo de edad $1(47,4 \mathrm{~g})$ de merluza común, como presa, presenta coeficientes de selectividad que se incrementan continuamente con la edad del predador, siguiendo en importancia el grupo de edad 2 (137 g) y luego el grupo de edad 3 (273 g). Los grupos de edad 4 y mayores presentan coeficientes de selectividad menores, siendo prácticamente nulo en merluzas de más de 5 años (Fig. 5). Asimismo, los coeficientes de selectividad indican que para la merluza predadora de 0 a 4 años de edad, otras presas son más importantes $(>29 \%)$, probablemente zooplancton (eufáusidos) y peces mesopelágicos como mictófidos.

En el caso de las presas langostino colorado y amarillo, la matriz de coeficientes de selectividad indica que prácticamente todo el rango de edades de estos crustáceos son preferidos por merluza común.
Los grupos de edad 0 y 1 de estas presas presentan sólo coeficientes de selectividad importante para merluza de 0 a 2 años de edad (Fig. 5).

En los clupeiformes sardina común y anchoveta, el grupo de edad 0 de estas presas (6-7 g) es más preferido por merluzas de 2 y más años, sin diferenciarse mucho el resto de los grupos de edad de la presa (Fig. 5). La anchoveta, en cambio, revela que la merluza común de 2 a 5 años prefiere presas del grupo de edad 0 y 1 , mientras que las merluzas de más de 5 años prefieren anchovetas de más de 1 año.

En el sistema bajo análisis, la merluza común es el único predador, de tal manera que la ración de alimento per cápita sólo se necesita para esta especie, cuyos resultados se presentan en la Tabla 4. Esta se incrementa con la edad del predador, en función del peso corporal, desde 0,063 a $6,38 \mathrm{~kg} \cdot a n ̃ o{ }^{-1}$. 

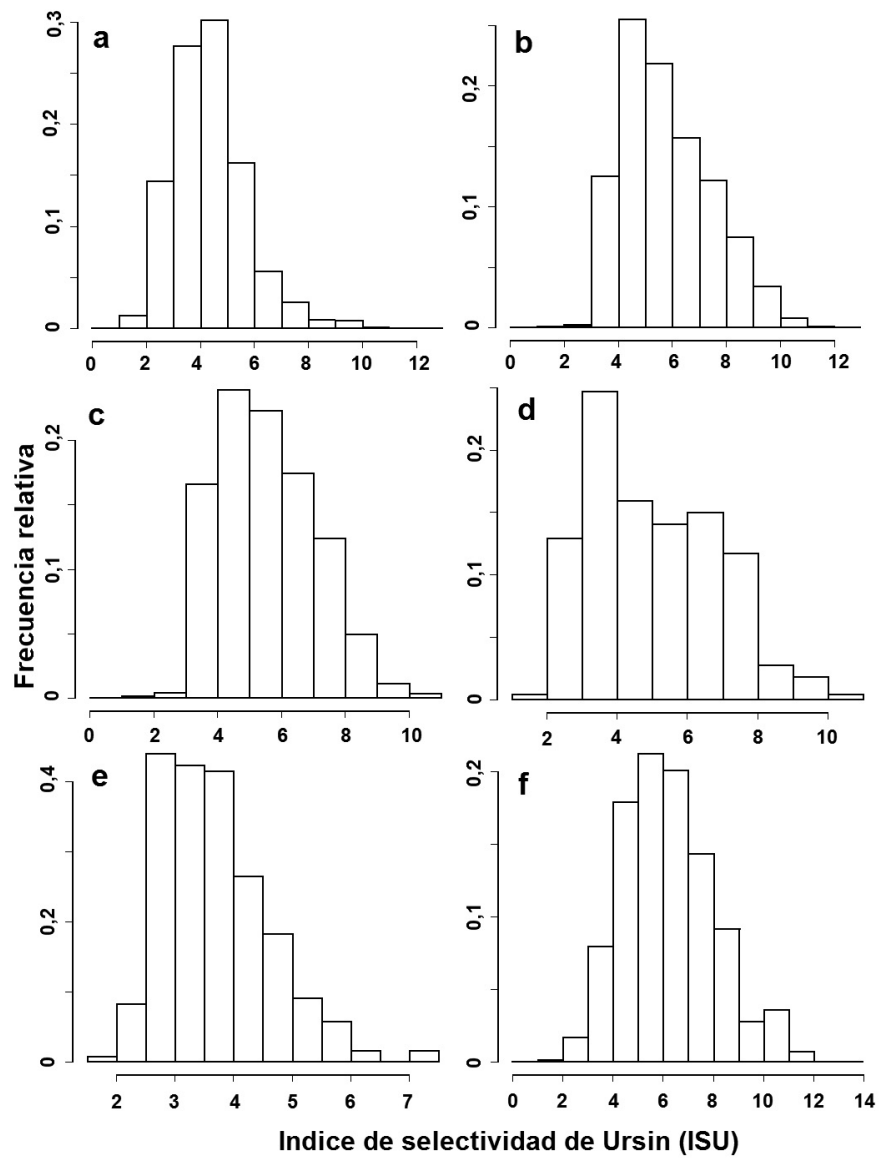

Figura 3. Histogramas de frecuencia del índice de selectividad de presa de Ursin para cada una de las presas en los contenidos estomacales de merluza común (Merluccius gayi gayi) en el periodo 1992-1997. a) merluza común (canibalismo), b) langostino colorado, c) langostino amarillo, d) sardina común, e) anchoveta, f) otras presas.

Figure 3. Frequency histograms of Ursin's prey selectivity index (ISU) for each prey in the stomach contents of Chilean hake (Merluccius gayi gayi) in the period 1992-1997. a) hake (cannibalism), b) red squat-lobster, c) yellow squat-lobster, d) common sardine, e) anchovy, f) other preys. 


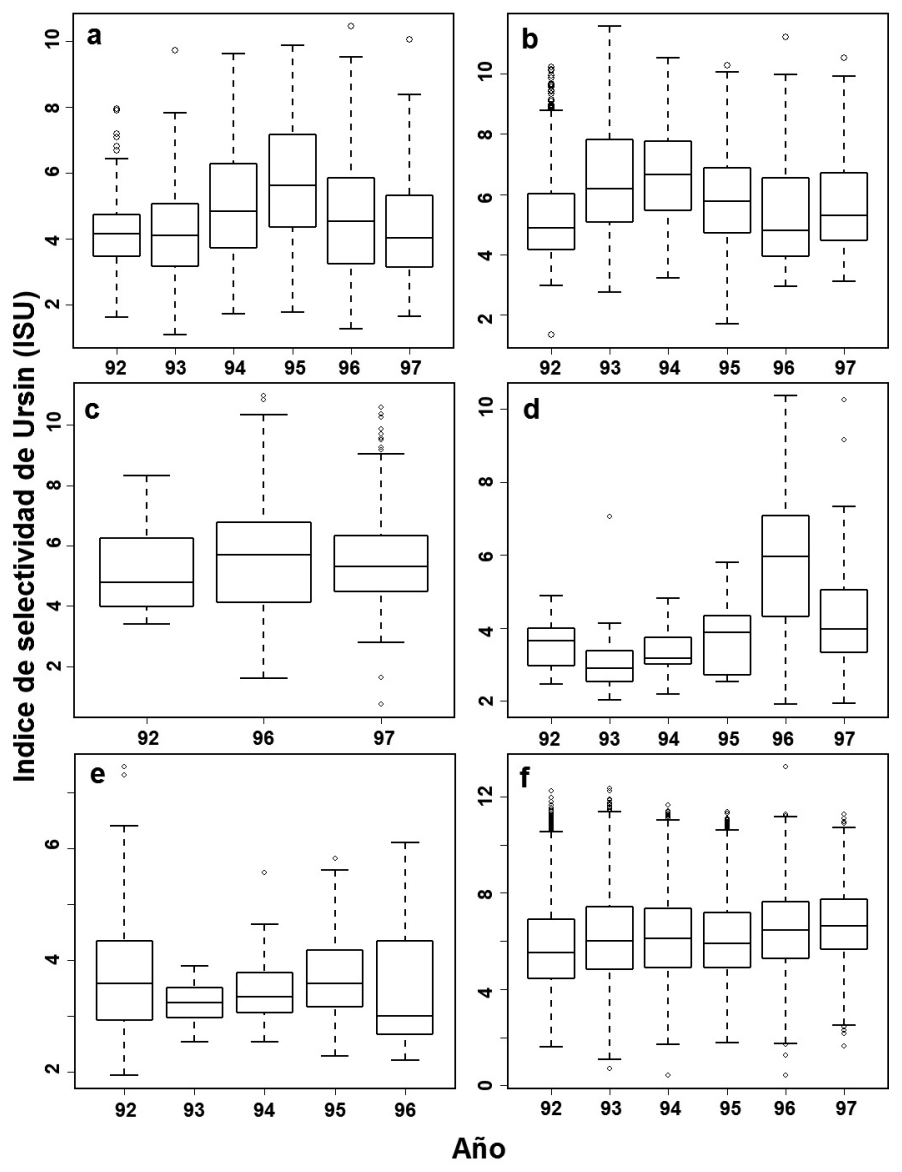

Figura 4. Gráficos de caja del índice de selectividad de presa de Ursin (ISU) para cada una de las presas por año. a) merluza común (canibalismo), b) langostino colorado, c) langostino amarillo, d) sardina común, e) anchoveta, f) otras presas.

Figure 4. Box-plots of Ursin's prey selectivity index (ISU) for each prey per year. a) hake (cannibalism), b) red squatlobster, c) yellow squat-lobster, d) common sardine, e) anchovy, f) other preys.

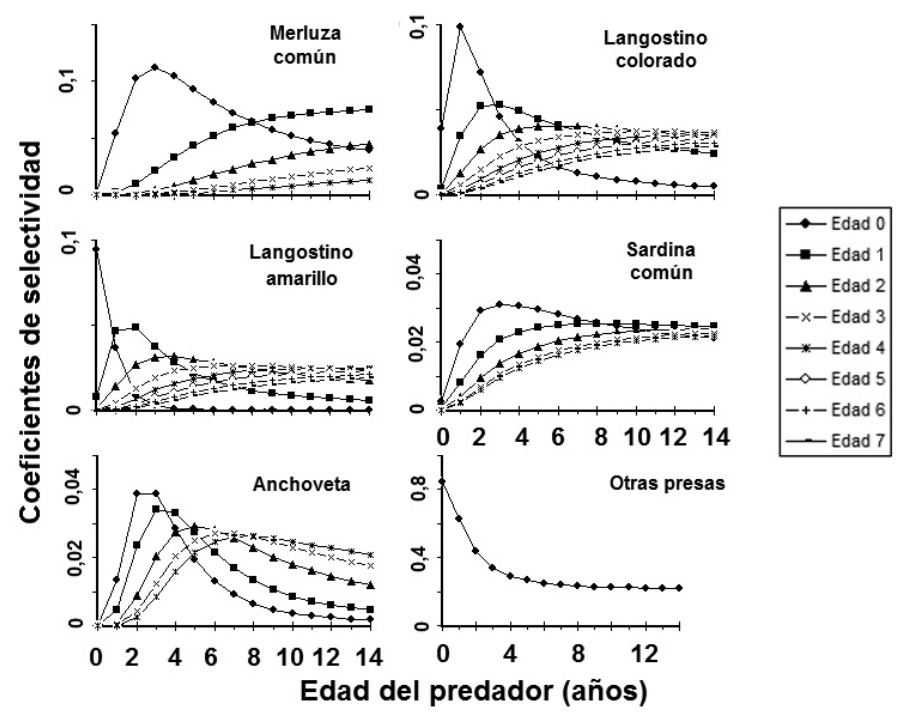

Figura 5. Coeficientes de selectividad para las presas de merluza común (Merluccius gayi gayi) en el periodo 1992-1997.

Figure 5. Prey selectivity coefficients for Chilean hake (Merluccius gayi gayi) preys in the period 1992-1997. 
Tabla 4. Peso promedio (W), tasa de crecimiento (dw/dt), eficiencia de conversión de alimento $\left(K_{1}\right)$ y ración de alimento per cápita $\left(\mathbf{R}_{\mathrm{x}, \mathrm{j}}\right)$ para merluza común por grupos de edad.

Table 4. Average weight $(W)$, growth rate $(d w / d t)$, food conversion efficiency $\left(K_{1}\right)$, and food ratio per capita $\left(R_{x, j}\right)$ for Chilean hake per age groups.

\begin{tabular}{|c|c|c|c|c|}
\hline $\begin{array}{l}\text { Edad } \\
\text { (años) }\end{array}$ & $\begin{array}{l}\text { W } \\
\text { (g) }\end{array}$ & $\begin{array}{c}d w / d t \\
\left(g \cdot a \tilde{n} 0^{-1}\right)\end{array}$ & $K_{1}$ & $\begin{array}{c}\mathbf{R}_{\mathrm{x}, \mathrm{j}} \\
\left(\mathrm{kg} \cdot \mathbf{a n ̃} 0^{-1}\right)\end{array}$ \\
\hline 0 & 6,4 & 19,5 & 0,309 & 0,063 \\
\hline 1 & 47,4 & 64,6 & 0,224 & 0,289 \\
\hline 2 & 136,9 & 114,1 & 0,174 & 0,655 \\
\hline 3 & 273,4 & 157,4 & 0,140 & 1,122 \\
\hline 4 & 448,3 & 190,5 & 0,115 & 1,653 \\
\hline 5 & 650,7 & 212,5 & 0,096 & 2,216 \\
\hline 6 & 869,9 & 224,5 & 0,081 & 2,788 \\
\hline 7 & 1096,8 & 228,0 & 0,068 & 3,349 \\
\hline 8 & 1323,7 & 224,9 & 0,058 & 3,889 \\
\hline 9 & 1544,9 & 216,9 & 0,049 & 4,398 \\
\hline 10 & 1756,5 & 205,6 & 0,042 & 4,871 \\
\hline 11 & 1955,5 & 192,2 & 0,036 & 5,307 \\
\hline 12 & 2140,5 & 177,7 & 0,031 & 5,705 \\
\hline 13 & 2310,7 & 162,7 & 0,027 & 6,065 \\
\hline 14 & 2465,9 & 147,9 & 0,023 & 6,389 \\
\hline
\end{tabular}

\section{DISCUSIÓN}

El índice de selectividad de presas por tamaños de Ursin (1973) asume que los predadores tienen una razón constante basada en la amplitud del nicho trófico, de tal manera que la selectividad y la preferencia de presas por tamaño es independiente del tamaño del predador. En este contexto, se está asumiendo un espectro de biomasa plano con una pendiente igual a cero y que la distribución de frecuencia de la razón de peso predador-presa describe una distribución de frecuencia log-normal para la selectividad de presas por tamaño. El máximo de la distribución marca la razón de peso predador-presa preferida, utilizándose el promedio como estimador de éste. De esta manera, el peso absoluto de la presa preferida se incrementa proporcionalmente con el peso absoluto del predador.

Los resultados obtenidos indican que el peso de las presas en los contenidos estomacales de merluza común se incrementa con el peso y tamaño del predador, situación que queda demostrada en el caso de la merluza común (canibalismo), langostino colorado, langostino amarillo, anchoveta, y otras presas, excepto en el caso de la sardina común. Las relaciones entre el peso de la presa y el tamaño de la presa no fueron estrictamente lineales, ya que a partir de los $1500 \mathrm{~g} \mathrm{o} 60 \mathrm{~cm}$ del predador el peso de las presas exhibe una tendencia a la estabilización. Este comportamiento sin duda se debe al tamaño máximo que pueden alcanzar las presas respecto del tamaño del predador, particularmente los langostinos y la anchoveta. En el caso de la merluza común, queda demostrado que el canibalismo es importante solo sobre ejemplares menores de 3-4 años que alcanzan un peso promedio de hasta $450 \mathrm{~g}$ aproximadamente. La excepción general fue encontrada en la sardina común, probablemente debido a cambios interanuales en la fracción de tamaños de la presa en los contenidos estomacales, tal como se observa en el índice de selectividad de Ursin calculado para esta especie (Tabla 2, Fig. 4). Dichos cambios interanuales podrían ser consecuencia de la variabilidad interanual de la abundancia de sardina común, afectando por ende la vulnerabilidad a la predación.

Para el periodo 1992-1997, se encontró evidencia de asimetría en la distribución de frecuencia del índice de Ursin. Sin embargo, la distribución log-normal ha sido utilizada tradicionalmente para medir la preferencia por tamaño a pesar de que existe evidencia de distribuciones de frecuencia asimétricas (Tsou \& Collie, 2001). Tal asimetría podría ser consecuencia de otros factores relativos al espectro de tamaños de las presas. Por ejemplo, Floeter \& Temming (2003, 
2005) demuestran que la razón de pesos predadorpresa preferida es una función exponencial que se incrementa con el tamaño del predador y una función exponencial decreciente de la pendiente del espectro de tamaño en número en el ambiente. Sin embargo, para evaluar el espectro de tamaño se requiere conocer la abundancia de las presas por tamaño en el mar, y por estrato geográfico, con el objeto de analizar coeficientes de selección del tipo Chesson (1978), y relacionar el peso de la presa contra la razón de pesos predador-presa.

Los resultados de este estudio confirman la hipótesis que a medida que los ejemplares crecen tienden a consumir presas de mayor tamaño corporal, pero en términos absolutos el efecto neto se debería traducir en el consumo de la fracción más juvenil de las poblaciones de presa. Al menos, esta afirmación es evidente en el caso del canibalismo sobre merluzas de 0 a 3 años de edad, y sobre ejemplares de anchoveta del grupo de edad 0 y 1 . En el caso del langostino colorado y langostino amarillo, la preferencia por tamaño (edad) de estas presas revela coeficientes de adecuación similar para todo el rango de edades potenciales de estos crustáceos. En efecto, solamente la fracción juvenil del predador (grupos de edad 0 a 2 años) seleccionan langostinos del grupo de edad 0 y 1 años. Sin embargo, en la fracción juvenil del predador, "otras presas" como eufáusidos y mictófidos son más importantes debido a cambios ontogenéticos en la dieta (Arancibia et al., 1998).

En relación con la ración de alimento per cápita, Jurado-Molina et al. (2006) utilizaron la tasa de crecimiento dividida por la tasa de eficiencia gruesa de conversión de alimento ad hoc basada en relaciones empíricas establecidas por Brett \& Groves (1979) para peces, y estimaron $0,83 \mathrm{~kg} \cdot \mathrm{año}^{-1}$ para merluzas de 3 años de edad, 1,09 $\mathrm{kg} \cdot$ año $^{-1}$ para la edad 4, y

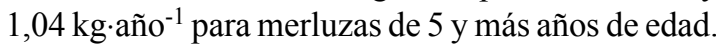
Estas tasas aparecen subestimadas respecto de las calculadas en este estudio, y basadas en una ración diaria de $6,03 \mathrm{~g} \cdot \mathrm{dia}^{-1}$ y la eficiencia de conversión de alimento establecida por Cubillos et al. (2003b), quienes además estimaron una razón consumo-biomasa (Q/B) de 4,98 veces su propio peso.

En este estudio se determina por primera vez los coeficientes de selección o preferencia para las presas de la merluza común con información de contenidos estomacales, y orientados al análisis multiespecífico basado en interacciones tróficas. El objetivo radica en su potencialidad para el desarrollo de futuras aplicaciones basadas en los supuestos del Análisis de Población Virtual Multiespecífico, entre otras aproximaciones recientes (e.g. Flexibest, Frøysa et al., 2002; GADGET, Bogstad et al., 2003) que pueden ser utilizadas para evaluar la mortalidad por predación ejercida por la merluza común. Una de las debilidades de esta aproximación radica en que la preferencia de presas no es una función de la abundancia de las presas o de otras variables en el MSVPA (Magnusson, 1995). En otras palabras no hay oportunidad para que el predador cambie sus preferencias por presas alternativas en respuesta a los cambios en la abundancia de las presas. La ración es constante para el predador y, por lo tanto, no puede cambiar su nivel de alimentación en respuesta a los cambios en abundancia de las presas. No obstante, el supuesto de constancia en los coeficientes de adecuación o preferencia de las presas parece ser razonable según Rice et al. (1991). En consecuencia, las bases cuantitativas aquí desarrolladas permitirán avanzar en la evaluación de la mortalidad por predación de langostinos, clupeiformes y canibalismo por merluza común, el cual constituye el predador más importante en el sistema bento-demersal y pelágico de Chile centro-sur.

\section{AGRADECIMIENTOS}

Los autores agradecen al Fondo de Investigación Pesquera la autorización para publicar los resultados del Proyecto FIP 2004-43 "Evaluación de la mortalidad por predación de la merluza común sobre langostino colorado y langostino amarillo, y canibalismo". Luis Cubillos agradece los comentarios y sugerencias de tres revisores anónimos del manuscrito.

\section{REFERENCIAS}

Aguayo, M. \& V. Ojeda. 1987. Estudio de la edad y crecimiento de merluza común (Merluccius gayi gayi Guichenot, 1848) (Gadiformes-Merluccidae). Invest. Pesq. (Chile), 34: 99-112.

Angelescu, V. \& L.B. Prenski. 1987. Ecología trófica de la merluza común del mar Argentino (Merlucciidae, Merluccius hubbsi). Parte 2. Dinámica de la alimentación analizada sobre la base de las condiciones ambientales, la estructura y las evaluaciones de los efectivos en su área de distribución. Contrib. Inst. Nac. Invest. Des. Pesq. (INIDEP), Mar del Plata, 561: 205 pp.

Arana, P. \& S. Williams. 1970. Contribución al conocimiento del régimen alimentario de la merluza 
(Merluccius gayi). Invest. Mar., Valparaíso, 1(7): 139-154.

Arancibia, H. 1989. Distribution patterns, trophic relationships and stock interactions in the demersal fish assemblage off central Chile. Dr. rer. nat. Tesis. University of Bremen, RFA, 221 pp.

Arancibia, H., J. Toro, V. Fernández \& R. Meléndez. 1986. Estimación de la mortalidad por depredación del langostino colorado (Pleuroncodes monodon) por la merluza común (Merluccius gayi) en el área $35^{\circ} 45^{\prime}$ S- $37^{\circ} 10^{\prime}$ S. En: P. Arana (ed.). La pesca en Chile. Escuela de Ciencias del Mar, Universidad Católica de Valparaíso, Valparaíso, pp. 57-67.

Arancibia, H. \& R. Meléndez. 1987. Alimentación de peces concurrentes en la pesquería de Pleuroncodes monodon Milne Edwards. Invest. Pesq. (Chile), 34: 113-128.

Arancibia, H. \& M. Fuentealba. 1993. Análisis de la alimentación de Merluccius gayi gayi (Guichenot, 1848) de Chile central, en el largo plazo. Biol. Pesq., 22: 5-11.

Arancibia, H., M. Catrilao \& B. Farías. 1998. Evaluación de la demanda de alimento en merluza común y análisis de su impacto en pre-reclutas. Informe Final Proyecto FIP $N^{\circ}$ 95-17, Universidad de Concepción, Fondo de Investigación Pesquera (FIP), Informe Técnico IT/95-17: 93 pp.

Arancibia, H., S. Neira, V. Christensen, R. Olson, F. Arreguín-Sánchez, L. Cubillos, R. Quiñones, C. Gatica \& M. Medina. 2003. Enfoque metodológico para el análisis ecosistémico en la administración de pesquerías de la zona central de Chile. Inf. Téc. FIP-IT/ 2001-29: 278 pp.

Arancibia, H., L.A. Cubillos \& E. Acuña. 2005. Annual growth and age composition of the squat lobster Cervimunida johni off northern - central Chile (1996-97). Sci. Mar., 69(1): 113-122.

Bahamonde, N. \& G. Cárcamo. 1959. Observaciones sobre la alimentación de la merluza (Merluccius gayi) en Talcahuano. Invest. Zool. Chil., 5: 211216.

Beyer, J. \& P. Sparre. 1983. Modelling exploited marine fish stocks. En: S.E. Jørgensen (ed.). Application of ecological modelling in environmental management, Part A. Elsevier Scientific Publishing, Amsterdam, pp. 485-582.

Bogstad, B., D. Howell, K.G. Frøysa, M.N. Asnes \& J. Begley. 2003. Modelling survival and growth in age- and length-structured population dynamics models, and relating model variables to observations in such models. Working document,
ICES Study Group on age-length structured assessment models (SGASAM), Bergen, Norway, June 3-6 2003, 25 pp.

Brett, J.R. \& T.D.D. Groves. 1979. Physiological energetics. En: W.D. Randall \& J. Brett (eds.). Bioenergetics and Growth, Fish Physiology, vol 8. Academic Press, New York, pp. 279-352.

Canales, M., J.C. Saavedra, G. Böhm \& A. Aranis. 2004. Investigación CTP de sardina común, zona centro-sur. Informe Final. IFOP/SUBPESCA, 41 pp.

Canales, T.M. \& C. Canales. 2005. Investigación captura total permisible anchoveta centro-sur 2005. Informe Final IFOP/SUBPESCA, 41 pp.

Castillo, R., L. Juárez \& L. Higgison. 1989. Predación y canibalismo en la población de la merluza peruana de la zona de Paita. En: Memorias del Simposio Internacional sobre recursos vivos y pesquerías en el Pacífico Sudeste (Viña del Mar, Chile, 1988). Rev. Com. Perm. Pacífico Sur (Número especial), pp. 273-286.

Chesson, J. 1978. Measuring preference in selective predation. Ecology, 59: 211-215.

Christensen, V. \& D. Pauly. 1992. ECOPATH II: a software for balancing steady-state ecosystem models and calculating network characteristics. Ecol. Model., 61: 169-185.

Clark, M.R. 1985. The food and feeding of seven fish species from the Campbell Plateau, New Zealand. J. Mar. Freshwater. Res., 19: 339-363.

Cubillos, L.A., D. Arcos \& A. Sepúlveda. 2003a. La pesquería chilena de merluza común: ¿Es suficiente el uso de modelos cuantitativos de evaluación para asesorar científicamente al manejo de la pesquería? En: E. Yáñez (ed.). Actividad pesquera y de acuicultura en Chile. Escuela de Ciencias del Mar, Universidad Católica de Valparaíso, Valparaíso, pp. 209-220.

Cubillos, L.A., H. Rebolledo \& A. Hernández. 2003b. Prey composition and estimation of $Q / B$ for the Chilean hake, Merluccius gayi (GadiformesMerluccidae), in the central-south area off Chile (34은 $-40^{\circ}$ S). Arch. Fish. Mar. Res., 50(3): 271-286.

Espino, M. \& V. Wosnitza-Mendo. 1989. Relación entre las poblaciones de merluza (Merluccius gayi peruanus) y anchoveta (Engraulis ringens). En: R. Jordán, R. Kelly, O. Mora, A.Ch. de Vildoso \& N. Henríquez (eds.). Memorias del Simposio Internacional de los Recursos Vivos y Pesquerías del Pacífico Sudeste. Rev. Com. Perm. Pacífico Sur (Número especial), pp. 267-271. 
Floeter, J. \& A. Temming. 2003. Explaining diet composition of North Sea cod (Gadus morhua): prey size preference vs. prey availability. Can. J. Fish. Aquat. Sci., 60: 140-150.

Floeter, J. \& A. Temming. 2005. Analysis of prey size preference of North Sea whiting, saithe, and grey gurnard. ICES J. Mar. Sci., 62: 897-907.

Frøysa, K.G., B. Bogstad \& D.W. Skagen. 2002. Fleksibest - an age-length structured fish stock assessment model. Fish. Res., 55: 87-101.

Fuentealba, M. 1993. Relaciones tróficas intraespecíficas de la merluza común, Merluccius gayi (Guichenot, 1848); de Chile Central. Tesis Magíster en Ciencias (Zoología), Universidad de Concepción, Concepción, 110 pp.

Gallardo, V.A., H. Bustos, A. Acuña, L. Díaz, V. Erbs \& R. Meléndez. 1980. Relaciones ecológicas de las comunidades bentónicas y bentodemersal de la plataforma continental de Chile central. Informe Final. Convenio Subsecretaría de Pesca / Universidad de Concepción, 325 pp.

Hermosilla, I. 1959. Contribución al estudio del régimen alimentario de Merluccius gayi (Guichenot) frente a la zona de Concepción. Memoria de Profesor de Biología. Universidad de Concepción, Concepción, $50 \mathrm{pp}$.

Hollowed, A.B., J.N. Ianelli \& P.A. Livingston. 2000. Including predation mortality in stock assessments: a case study for Gulf of Alaska walleye Pollock. ICES J. Mar. Sci., 57: 279-293.

Hoyl, A. 1967. Alimentación de la merluza Merluccius gayi (Guichenot) en la zona de Valparaíso (Chile). Memoria de Técnico en Industrias Pesqueras. Universidad Católica de Valparaíso, Valparaíso, 25 pp.

Ihaka, R. \& R. Gentleman. 1996. R: A language for data analysis and graphics. J. Comput. Graph. Stat., 5: 299-314.

Jurado-Molina, J., C. Gatica \& L. Cubillos. 2006. Incorporating cannibalism into age-structured model for the Chilean hake. Fish. Res., 82: 30-40.

Livingston, P. \& K. Bailey. 1985. Trophic role of the Pacific whiting, Merluccius productus. Mar. Fish. Rev., 47(2): 16-22.

Livingston, P.A., D.A. Dwyer, D.L. Wencker, M.S. Yang \& G.M. Lang. 1986. Trophic interactions of the key fish species in the Eastern Bering Sea. Inst. North. Pacific. Fish. Comm. Bull., 47: 49-65.

Livingston, P.A. \& J. Jurado-Molina. 2000. A multispecies virtual population analysis of the eastern Bering Sea. ICES J. Mar. Sci., 57: 294299.

Macpherson,E. \& B. Roel. 1987. Trophic relationships in the demersal fish community off Namibia. En: A.I.L. Payne, J.A. Gulland \& K.H. Brink (eds.). The Benguela and comparable ecosystems. S. Afr. J. Mar. Sci., 5: 585-596.

Magnusson, K. 1995. An overview of the multispecies VPA - theory and applications. Rev. Fish Biol. Fish., 5: 195-212.

Meléndez, R. 1984. Alimentación de Merluccius gayi (Guichenot) frente a Chile central $\left(32^{\circ} 05^{\prime} \mathrm{S}\right.$ $36^{\circ} 50^{\prime}$ 'S). Bol. Mus. Nac. Hist. Nat., Chile, 54 pp.

Neira, S., H. Arancibia \& L. Cubillos. 2004. Comparative analysis of trophic structure of commercial fishery species off Central Chile in 1992 and 1998. Ecol. Model., 172: 233-248.

Pauly, D. 1986. A simple method for estimating the food consumption of fish populations from growth data of food conversion experiments. Fish. Bull., 84: 827-840.

Payne, A., B. Rose \& W. Leslie. 1987. Feeding of hake and a first attempt at determining their trophic role in the South African west coast marine environment. En: A.I.L. Payne, J.A. Gulland \& K.H. Brink (eds.). The Benguela and comparable ecosystems. S. Afr. J. Mar. Sci., 5: 471-501.

Punt, A., R. Leslie \& S. Du Plessis. 1992. Estimation of the annual consumption of the food by Cape hake Merluccius capensis and M. paradoxus off the South African west coast. Benguela trophic functioning. S. Afr. J. Mar. Sci., 12: 611-634.

Rice, J.C., N. Daan, J.G. Pope \& H. Gislason. 1991. The stability of estimates of suitabilities in MSVPA, over 3 years of data from predator stomachs. ICES Mar. Sci. Symp., 193: 34-45.

Roa, R. 1993. Annual growth and maturity function of the squat lobster Pleuroncodes monodon in central Chile. Mar. Ecol. Prog. Ser., 97: 157-166.

Stobberoup, K. 1992. Food composition and consumption of Chilean hake (Merluccius gayi G.) with special reference to cannibalism. ICES Counc. Meet. Pap./G 43: 30 pp.

Tsou, T.-S. \& J.S. Collie. 2001. Estimating predation mortality in the Georges Bank fish community. Can. J. Fish. Aquat. Sci., 58: 908-922.

Ursin, E. 1973. On the prey size preference of cod and dab. Meddr. Danm. Fisk.-og Havunders., 7: 85-98.

Velasco, F. \& I. Olaso. 1998. European hake Merluccius merluccius (L., 1758) feeding in the 
Cantabrian Sea: seasonal, bathymetric and length variations. Fish. Res., 32: 33-34.

Walters, C., V. Christensen \& D. Pauly. 1997. Structuring dynamic models of exploited ecosystems from trophic mass-balance assessments. Rev. Fish Biol. Fish., 7: 139-172.

Wipple, S.J., J.S. Link, L.P. Garrison \& M.J. Fogarty. 2000. Models of predation and fishing

Recibido: 4 octubre 2006; Aceptado: 9 abril 2007 mortality in aquatic ecosystems. Fish. Fish., 1: 2240.

Wood, S.N. 2000. Modelling and smoothing parameter estimation with multiple quadratic Penalties. J. R. Stat. Soc. B, 62: 413-428.

Wood, S.N. 2003. Thin-plate regression splines. J. R. Stat. Soc. B, 65: 95-114. 